\title{
An Efficient Approach for Content-Based Image Retrieval Using Cuckoo Search Optimization
}

\author{
Palwinder Kaur and Rajesh Kumar Singh
}

\begin{abstract}
The CBIR is a process that retrieves images on the basis of shape, texture and color, etc that is basically features. It works by retrieving images related to Query Image (QI) from big databases. In existing CBIR systems retrieval efficiency is confined by extracting limited feature sets. Different optimization algorithms like Particle swarm optimization, Genetic algorithm, etc have been used for optimization purpose which are very old algorithms. There are number of other algorithms that have been proposed after it that gives better optimized results. Afterwards there is need of classifier to retrieve exact set of the images that will increase the performance of the proposed system and meets the user's requirement. So, in this paper we have proposed a new approach in which firstly image content is improved using Clahe then features are extracted using independent component analysis and select the relevant features that reduces the semantic gap by learning discriminative features directly from the images. Afterwards trained distinct medical image selected features from dataset are optimized using Cuckoo search to improve the accuracy and precision values. The proposed system performance is improved using Support vector machine classifier which meets the user expectation and increase the efficiency of the system. The proposed system is tested in MATLAB in terms of recall, Precision, etc various parameter. The DICOM dataset is used for testing purpose.
\end{abstract}

Index Terms-CBIR, DICOM, CUCKOO, CLACHE, independent component analysis.

\section{INTRODUCTION}

Due to advances in image acquisition and data storage there is need to create large data sets. The need for keeping these databases is growing because of the increasing amount of digitally produced images in areas like medicine, journalism, and private life. There databases should be managed in efficient way [1]. In computer vision the process of desired images retrieval from image database in an efficient way is very important. It gives convenience and ease to users due to which its use is getting increased in different fields. Number of retrieval systems has been proposed by different researchers to achieve content based image retrieval. Text and content based retrieval are two frameworks that are used for performing information retrieval. In 1970s Text based framework was introduced in which image is annotated by text descriptors that are used in database for information retrieval but it has some disadvantages such as more human work is required to annotate the images and annotation inaccuracy.
So, in 1980s Content-based image retrieval (CBIR) is introduced to overcome the text based framework problems. In this approach image contents are used to annotate to perform retrieval. The term "CONTENT" might refer to low level feature such as shape, color, texture, or any other important information derived from the image [2]. "Content-based" means analyzing the image contents not the text descriptors such as tags, descriptions or keywords correlated with image. The extraction of features or contents of image is basic function of CBIR. The main difference between both is that there is need of human interaction in case of text based system [2]. Keywords, descriptors or high level features and text are used by humans for getting images information and for find out their similarity. Available computer vision techniques can be used for automatically extraction of image features that are mostly low level features. By extracting these features, searching, browsing from a database and similarity matching between the images is performed [2]-[7]. Image features are used in case of CBIR system instead of image itself that makes its most important advantage.

In beginning mostly used image retrieval systems is QBIC system from IBM [3]. Photobook system from Massachusetts Institute of Technology (MIT) [4] is also one of such systems. UC Berkley developed Blobworld is another image retrieval system [5]. In medical field a great importance has been played by CBIR as every day large amounts of images are accessed by doctors. In order to deal with those images kept in large databases need efficient and quick method [5]. Journalism is another field of life in which image saving and retrieving is of great importance. Journalists need to search images by new techniques that give more efficient and accurate results [6]. There are various websites where photos are shared that gives easiness to user for storing their images and allow others to view it.

This complete paper is divided into different sections in which first section gives brief introduction about image retrieval and their types. The second section focus on previous work done in this field and problem related to it along with how we reduce it using proposed approach is given in third section. The fourth section gives detail of experiment setup and simulation next section gives results obtained using proposed approach and their effects in terms of different parameters. The last section ended the complete paper by giving conclusion obtained from survey and results.

\section{LITERATURE REVIEW}

Different problems associated to Content-Based Image Retrieval, CBIR system designing and their solution are the
Manuscript received February 24, 2019; revised April 12, 2019.

The authors are with R Inder Kumar Gujral Punjab Technical University, Kapurthala, Punjab, India (e-mail: palavibhangu@gmail.com). 
main motive of K. Kranthi Kumar et al., (2010) [8]-[11]. It outlines the problem, the proposed solution, the final solution and the accomplishments achieved. The need of CBIR development is arise due to increase in sizes of image database and it's used in various applications. In this paper first section shape, color and texture like image features are described that are extracted and used on the basis of similarity between images [12]-[16]. There are number of algorithms that can be use for feature extraction part that is also thoroughly explained by author. They have used MATLAB software in which first of all they have built a software application with image database the on comparison and retrieval basis images color and texture features are utilized. The use of large database has become possible due to increase in its usage with multimedia technology development.

The use of CBIR is proved as an effective method for image retrieval and management. In 2011, Guoyong Duan et al., [12] given some advantages of using Content-based image retrieval system and their key technologies. Compare to the shortcoming that only one feature is used in the traditional system, this paper introduces a method that combines color, texture and shape for image retrieval and shows its advantage [13]. They have also focused on various commonly used algorithms used for feature extraction and representation and image matching methods [17].

In computer vision applications a wide use of desired images retrieval from large collection has been found. There is need of accurate and efficient system to improve the retrieval performance [18]. The CBIR is a process that retrieves images on the basis of shape, texture and color, etc that is basically features. There are number of CBIR based approaches out of which three approaches comparison is given in Sumaira Muhammad Hayat Khan et al., (2012) [9] paper on the basis of similarity measures, image features that are taken for two images similarity. In image retrieval system an important image feature selection and calculating through meaningful way is very important. There is need to consider important features while feature vector constructing and for calculating distance between two feature vectors there is need to measure proper similarity [14].

A wide attention has been gain by CBIR from last few years. CBIR is closer to human semantics, in the context of image retrieval process. In historical search, medical images, surveillance, remote sensing and weather forecasting like applications a great use of CBIR has been found. In 2014, Roshi Choudhary, et.al [10] has proposed an image retrieval technique on the basis of content that extracts both texture as well as color features. On color images a color moment is used for extracting color features and on gray scale image a local binary pattern is used for extracting texture features. After that, a single feature vector is made by combining both texture and color features. Euclidian distance is used in the end for similarity matching purpose that compares database images feature vector with query images. They have used LBP for natural images but mainly its use has been found in face recognition. This combined approach provides accurate, efficient, less complex retrieval system.

Only limited feature sets are extracted by available CBIR systems that confine the efficiency of retrieval. In 2017, Mutasem K. Alsmadi et al., [13], proposed a system in which extensive robust and important features were extracted from the images database and then stored in the feature repository. The color texture features and color signature with the shape makes a feature set. The given QI in the similar fashion extract the features and a meta-heuristic algorithm called a memetic or genetic algorithm is used for achieving similarity between QI features and database images features [15]. Number of images is considering for making dataset and precision-recall value is calculated for evaluating an efficiency of the system.

\section{Problem Statement AND Proposed Work}

The CBIR is a process that retrieves images on the basis of shape, texture and color, etc from a large database. These selected features are used for evaluating the performance of CBIR system. The images are first represented in terms of features in a high dimensional feature space then similarity among images stored in the database that of a query image is measured in the feature space by using a distance metric e.g., Euclidean distance. Image data representation in terms of selecting similarity measure and features are considered being very critical components for CBIR systems. The goal of an image retrieval system is to retrieve a set of images from a collection of images such that this set meets the user's requirements. In historical archives, digital library, medical imaging, finger print identification, crime prevention and biodiversity information system those databases are used.

The content based and label based image retrieval are two categories of methods used for image retrieval. In label based method marked labels are used to measure the similarity between images which is lack of concern for the semantic information. For image representation an image content features used in CBIR methods which enable those complicated and unmarked image data to be quantified and retrieved.

In existing CBIR systems retrieval efficiency is confined by extracting limited feature sets. In the existing system author have used Genetic algorithm for optimization purpose which is very old algorithm there are number of other algorithms that has been proposed after it that gives better optimized results. Afterward there is need of classifier to retrieve exact set of the images that will increase the performance of the proposed system and meets the user's requirement.

In proposed work we will use DICOM, CT and MRI like medical datasets for training images for image retrieval system. Then extract and select the relevant features using independent component analysis that reduces the semantic gap by learning discriminative features directly from the images. Afterward trained distinct medical image selected features from dataset are optimized using Cuckoo search to improve the accuracy and precision values. The proposed system performance is improved using Support vector machine classifier which meets the user expectation and increase the efficiency of the system. The proposed system is tested in MATLAB in terms of recall, Precision, etc various parameter.

The objective behind this work is to analyze and study the existing Content based image retrieval system and use DICOM and other medical datasets for training image retrieval system image. The other objective is to extract and 
select the relevant features based on the feature extraction mechanism which further can be used to reduce the semantic gap by learning discriminative features directly from the images. The selected features are optimized using hybrid optimization algorithm that helps in improving precision and accuracy values.

\section{EXPERIMENTAL SETUP AND SIMULATION}

The below Fig. 1 Shows the GUI panel in which the user interface controls are used and also the user is able to attract the machine. In the below figure two main buttons are used one for testing and one for training. Firstly the user will click on the training button to train all the categories.

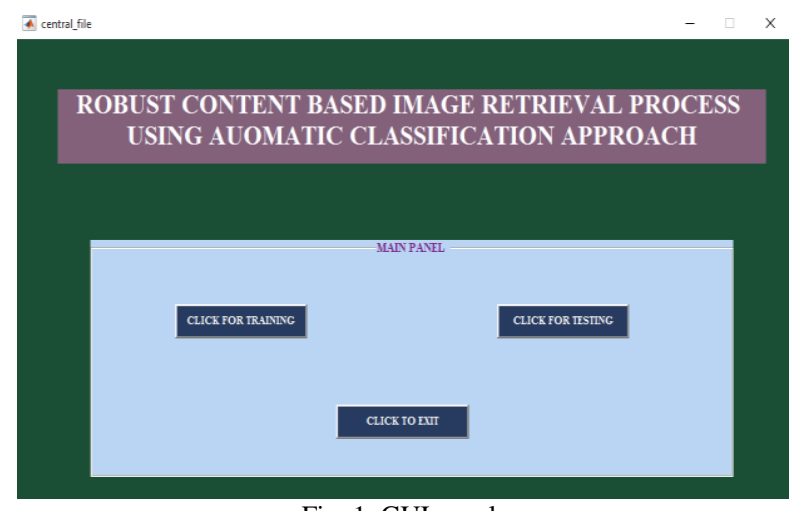

Fig. 1. GUI panel.

The below Fig. 2 shows the training panel in which the various pushbuttons are used and also the uploading of the categories are taken place. Each pushbutton consists of the training of the category which deals with the extraction and uploading of the input samples from dataset and also the feature optimization using cuckoo search.

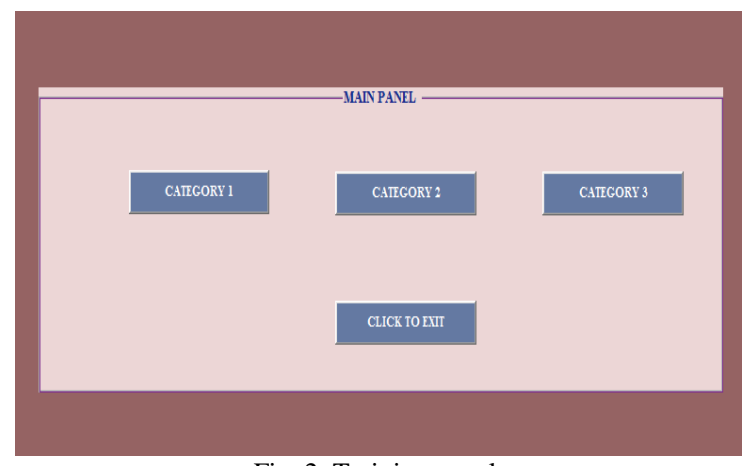

Fig. 2. Training panel.

When we click on above given category button to see resultant of the applied operations then a new window will be popup as shown in below Fig. 3 which shows an operative buttons that gives results scenarios.

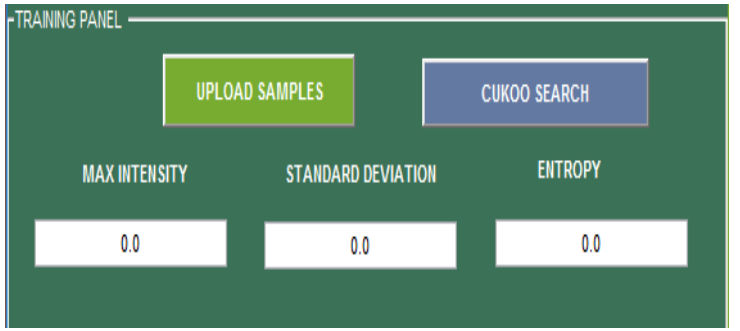

Fig. 3. Operative buttons.

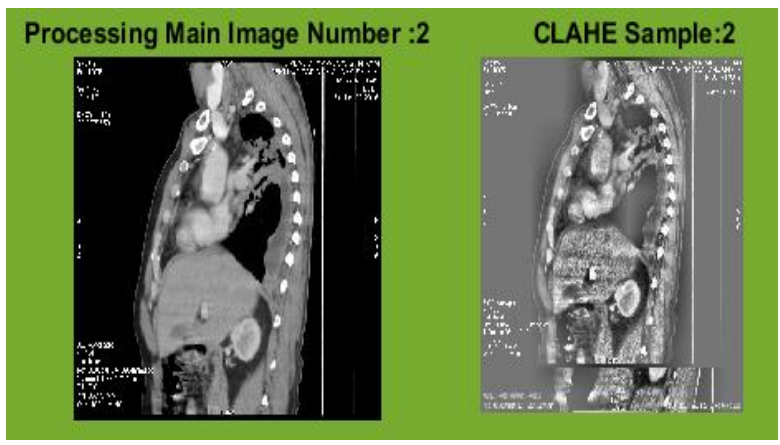

Fig. 4. Uploading panel.

The above Fig. 4 shows the category uploading and its resultant image as CLAHE which is named as contrast level image enhancement which deals with the adaptive equalization. It will enhance the contrast levels of the image and equalize the neighborhood pixels of the uploaded image as shown in above figure.

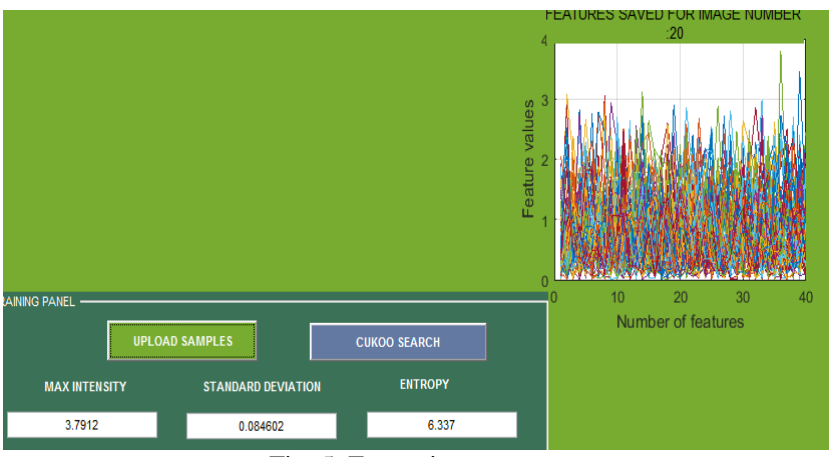

Fig. 5. Extraction process.

Fig. 5 shows the extraction of the image which is based on feature extraction process using independent component analysis (ICA) and also the feature vector is obtained which is shown in the graphical manner. Also the characteristics of the image will be evaluated in terms of maximum intensity of the image, standard deviations and entropy of the image.

ICA is a computational method to get hidden values of random variables. ICA basically designed for multivariate data. ICA is somewhat related to Principal Component Analysis (PCA). But it is capable when PCA fails. The data used for analyzing using ICA can be originated from many fields like economics, digital images, document databases etc.

ICA Algorithm Steps:

1) Make data to mean zero

2) Choose the number of components.

3) Whiten the data.

4) Choose random matrix.

5) Orthogonalised the matrix.

6) Do converged.

7) Repeat again.

8) Stop.

Fig. 6 shows the optimization of the features using instance selection approach and also the relevant feature vector which is extracted from the feature vector. The optimization is done using Cuckoo optimizer. This completes the training process and now the next step is to move to the testing phase.

Cuckoo search is an intelligent search that deals with the optimization algorithm which is inspired by the brood parasitism of various cuckoo species using lying of their spawns in the shells of other species or birds. Some birds can 
involve direct struggle with the interfering cuckoos. For instance, if a crowd bird determines the spawns are not their individually, it will throw these eggs far away or simply unrestraint its house and build a new house away. Some species have changed in such a method that female cuckoos are often actual specialized in the imitation in standards and decoration of the spawns of a few selected host species. Cuckoo search is flawless like breeding behavior which can be functional for numerous optimization difficulties.

\section{$\mathrm{CS}$ is based on three idealized rules:}

Each cuckoo lays one egg at a time, and dumps its egg in a randomly chosen nest

The best nests with high quality of eggs will carry over to the next generation.

The number of available host's nests is fixed, and the egg laid by a cuckoo is discovered by the host bird with a probability $(0,1)$. Discovering operates on some set of worst nests, and discovered solutions dumped from farther calculations.

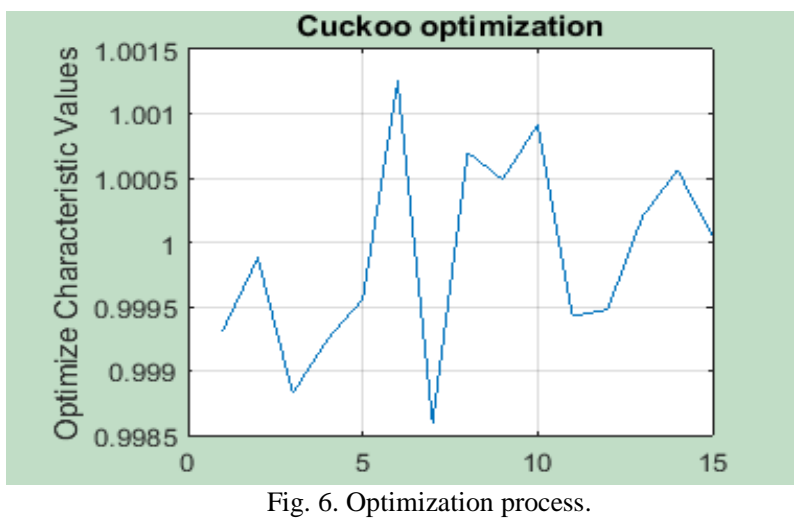

Fig. 7 shows the testing panel in which the random test image is uploaded and the same training process is applied on the test image as applied on all the three categories which gives the high similarity matched to the category of the training images.

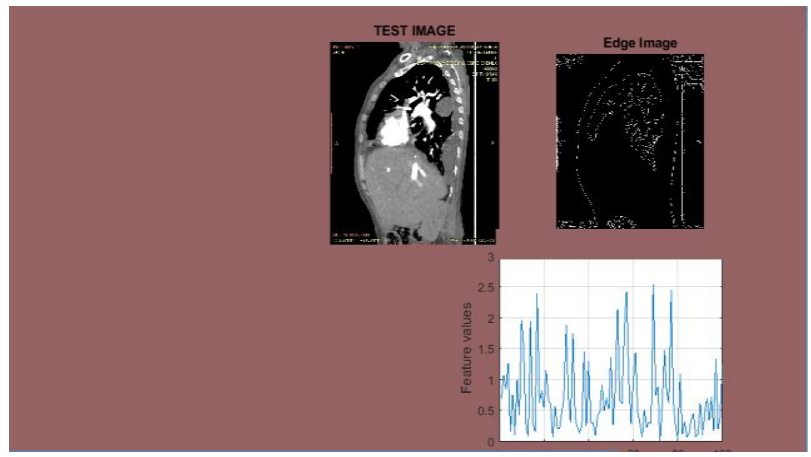

Fig. 7. Testing panel.

\section{RESUlTS AND DISCUSSION}

At the end we have used Support vector machine for Classification or testing the proposed approach. The below fig. 8 shows the testing result in terms of the classification which is done using Support vector machines and is able to perform the high classification results based on the training dataset.

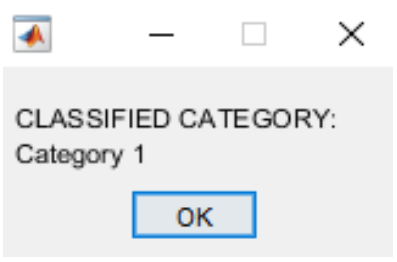

Fig. 8. Classified category.

The proposed approach is tested in terms of different parameters. Precision or sensitivity is a measure of the system ability in retrieving only similar images to the query image.

Precision=Number of similar images retrieved/ Total number of retrieved images

The ability to measures of CBIR system in terms of number of retrieved image with their similar images in the database is a measure of recall rate.

Recall $=$ Number of similar image retrieved $/$ Total number of similar images in the database

Sensitivity: 1-FAR

Where FAR is a false acceptance rate that deals with how much your system is able to accept the wrong samples based on feature extraction which must be low.

TABLE I: PARAMETERS EVALUATION
\begin{tabular}{|l|c|}
\hline Parameter & Values \\
\hline Recall & 0.44289 \\
\hline Precision & 0.69305 \\
\hline Sensitivity & 0.7024 \\
\hline F-Measure & 0.69769 \\
\hline
\end{tabular}

The above shown table gives the performance evaluations results of proposed approach in terms of high sensitivity rate which shows the high true positive rate, high recall which is totally based on the recalling the training process to classify the things in the testing phase which also must be high, high precision rate and high F-measure. If these evaluations are high then the performance will automatically increases with less error rate probabilities

\section{CONCLUSION}

Content based image retrieval (CBIR) is a computer vision technique that gives a way for searching relevant images in large databases. This search is based on the image features like color, texture and shape or any other features being derived from the image itself. The performance of a CBIR system mainly depends on these selected features. In this paper a new approach is proposed that helps in improving the performance of existing CBIR system. We have used DICOM dataset in which firstly selected image contrast are improved using Contrast Level Adaptive histogram equalization (CLAHE).

Feature extraction is a main part of CBIR which is done using Independent Component Analysis (ICA) which is related to Principal Component Analysis (PCA). The extracted features are optimized using cuckoo search optimization algorithm that gives best features from all extracted features. For testing purpose we have used Support vector machine classifier that will classify the selected image 
is belongs from which category. The proposed approach is tested in terms of different parameters and results obtained for recall is 0.44289 , Precision is 0.69305 , Sensitivity is 0.7024 and 0.69769 is the achieved F-measure using proposed approach.

\section{ACKNOWLEGMENT}

This work is supported by the Ik Gujral Punjab Technical University. We are very thankful to the University for the support and for providing us with the quality research papers for literature review.

\section{REFERENCES}

[1] T. Deselaers, "Image retrieval, object recognition, and discriminative models," Doctoral dissertation, December 2008.

[2] M. Kan, D. Xu, S. Shan, and X. Chen, "Semi supervised hashing via kernel hyper plane learning for scalable image search," IEEE Transactions on Circuits and Systems for Video Technology, vol. 24, no. 4, pp. 704-713, 2014.

[3] R. P. Picard and S. Sclaro, "Photobook: Content-based manipulation of image databases," International Journal of Computer Vision, vol. 18, pp. 233-254, 1996.

[4] Carson et al., "Blobworld: Image segmentation using expectation maximization and its application to image querying," IEEE Transaction on Pattern Analysis and Machine Intelligence, vol. 24, pp. 1026-1038, 2002.

[5] W. Muller, S. Muller, M. Maillet, and D. M. Squire, "Strategies for positive and negative relevance feedback in image retrieval," in Proc. International Conference on Pattern Recognition, vol. 1, pp. 1043-1046, Barcelona, Spain, Sept. 2000.

[6] H. Armitage and P. G. Enser. "Analysis of user need in image archives," Journal of Science, vol. 23, pp. 287-299, 1997.

[7] J. L. Shih and L. H. Chen, "Color image retrieval based on primitives of color moments," IEE E Proceedings- Vision Image and Signal Processing, vol. 149, pp 370-376, 2002.

[8] Y. Liu, D. S. Zhang, G. J. Lu, and W. Y. Ma, "A survey of content-based image retrieval with high-level semantics," The Journal of Pattern Recognition Society, vo1.40, pp. 262-282, 2007.

[9] S. M. H. Khan, A. Hussain and I. F. T. Alshaikhli, "Comparative study on content-based image retrieval (CBIR)," in Proc. 2012 International
Conference on Advanced Computer Science Applications and Technologies, pp. 61-66, 2012.

[10] R. Choudhary, N. Raina, N. Chaudhary, R. Chauhan, and R H. Goudar, "An integrated approach to content based image retrieval," in Proc. 2014 Internationai Conference on Advances in Computing, Communications and Informatics (ICACCI), pp. 2404-2410, 2014.

[11] K. K. Kumar and T. V. Gopal, "CBIR: Content based image retrieval," in Proc. National Conference on Advances in Information Security, pp. 1-6, 2010.

[12] G. Y, Duan, J. Yang, and Y. L. Yang, "Content-based image retrieval research," in Proc. 2011 International Conference Onphysics Science and Technology (ICPST 2011), pp. 471-477, 2011.

[13] M. K. Alsmadi, "An efficient similarity measure for content based image retrieval using memetic algorithm," Egyptian Journal of Basic and Applied Sciences, pp. 112-122, 2017.

[14] P. S. Nikkam and B. E. Reddy, "An efficient approach for content based image retrieval using hierarchical part-template and tree modeling," ICTACT Journal on Image and Video Processing, vol. 8, pp. 1607-1613, 2017.

[15] M. B. Suresh and B M. Naik, "An efficient approach of content based image retrieval using texture, color and shape features of an image," International Journal of Engineering Trends and Technology, vol. 48, pp. 316-320, 2017.

[16] J. Srinija, Y. Rajesh, and R. K. Tenali, "An efficient approach for content-based image retrieval using distributed networks," SSRG International Journal of Computer Trends and Technology, pp. 26-29, 2017.

[17] Faloutsos et al., "Efficient and effective querying by image content". Journal of Intelligent Information Systems, vol. 3, pp. 231-262, 1994.

[18] T. Y. Satheesha, D. Satyanarayana, M. N. G. Prasad, and K. D. Dhruve, "Melanoma Is skin deep: A 3D reconstruction technique for computerized dermoscopic skin lesion classification," IEEE Journal of Translational Engineering in Health and Medicine, vol. 5, pp. 1-17, 2017

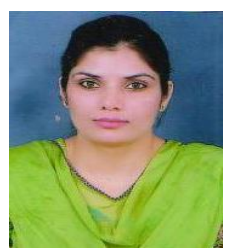

Palwinder Kaur is a research scholar from Inder Kumar Gujral Punjab Technical University, Kapurthala, Punjab, India. She has done M.Tech in Information \& Technology. She is currently working as Assistant Professor in KIMT, Ludhiana, India. Her research and publication interest include Medical informatics and optimization. 